\title{
La formación del profesional bibliotecario en el ámbito del Mercosur: realidad y tendencias en el inicio del siglo XXI
}

\author{
Ely Francina Tannuri de Oliveira \\ Universidade Estadual Paulista (Marília, Brasil)
}

\subsection{Resumen}

Se pretende analizar la formación inicial y continua del bibliotecario en los países del Mercosur con el fin de colaborar en el proceso de integración curricular. Tras contextualizar social y políticamente la evolución reciente de la educación en el Mercosur, se analizan los objetivos y organización curricular de las carreras, el perfil de los docentes y la oferta de cursos de educación continua. Se utilizaron metodologías propias del análisis documental y la Bibliotecología Comparada para analizar los programas del año 1996 y se realizó una encuesta sobre la titulación y régimen de contrato de los docentes y la oferta de formación continua. A partir de los resultados, se detectan los retos que se deben asumir en el proceso de convergencia educativa.

Palabras clave: Enseñanza profesional. Educación continua. Enseñanza reglada. Bibliotecología. Mercosur.

\subsection{Abstract}

Analysis of the Library and Information Science graduation and continued education courses in MERCOSUL countries in order to possibilitate their integration. After a contextualisation of the social and political evolution of education in the Mercosur, their curricula, teaching staff and continued education courses are considered. Documentary analysis was used for data gathering, and methods of Compared Librarianship and Compared Pedagogy were used for their analysis of curricula of the year 1996. Finally, the educational trends in librarian's education were assessed and the main challenges to be overcome in the process of convergence are stated.

Keywords: Mercosur. Librarianship. Continued education. Formal education. 


\section{Introducción}

Las intensas transformaciones económicas, sociales, políticas, culturales y filosóficas que marcaron las décadas de los 80 y 90 - especialmente la rearticulación del capitalismo y el agrupamiento de los países en grandes bloques regionales - ha producido cambios en todos los sectores, notablemente en la educación.

Cuestiones como la globalización, la información, las nuevas tecnologías, la calidad total, la calidad de enseñanza se han cruzado en el campo educativo y, por consiguiente, en la formación profesional en las diferentes áreas del conocimiento. Esta investigación pretende precisamente reflexionar sobre la formación del profesional bibliotecario en el ámbito del Mercosur y sobre las proyecciones del proceso de globalización en su formación. A partir de algunos presupuestos - a saber, el nuevo orden mundial marcado por la globalización de los mercados, el agrupamiento de los países en grandes bloques regionales, la internacionalización de los sistemas de enseñanza, la información considerada como elemento básico para el desarrollo social, económico y político, y las implicaciones entre transformaciones sociales y currículo-, esta investigación se propone cuatro objetivos: a) analizar, a la luz del nuevo orden mundial, las principales políticas públicas y los sistemas de educación superior en el ámbito del Mercosur; b) investigar la formación inicial del profesional bibliotecario, a través de estudio comparado de los diferentes currículos de los cursos del Mercosur; c) identificar el perfil de los docentes de los cursos de educación continua de Biblioteconomía de los tres últimos años, e investigar como se está implementando en las diferentes instituciones de enseñanza; y e) evaluar las tendencias educacionales en la formación del profesional en el cambio de siglo, señalando algunos desafíos en la formación del bibliotecario, analizando el efecto de la sociedad globalizada, sus problemas estructurales y las perspectivas para el futuro de la organización del trabajo. Se pretende, en suma, ofrecer datos y recomendaciones sobre la enseñanza de grado impartida en los países del Mercosur en el área de Biblioteconomía, facilitando la superación de las barreras existentes — debidas a las peculiaridades locales de los diferentes países - , la formación de una base académica única, y, en definitiva, la integración e internacionalización del sistema de educación superior. Específicamente, se pretende analizar los planes de estudio completos de los cursos de Biblioteconomía, y evaluar el perfil de los docentes, la oferta y la temática de los cursos de educación continua.

Respecto a la metodología, los planes de estudio se analizaron mediante la metodología del análisis documental para la recolección de datos, y la de la Biblioteconomía Comparada - que, por su parte, utiliza técnicas de otras áreas de las Ciencias Sociales, especialmente de la Pedagogía Comparada- para su estudio. La muestra estudiada consistió en un corte sincrónico realizado para el año 1996 que consideró, por primera vez, los siete cursos de la Argentina, treinta

Scire. $9: 1$ (en.-jun. 2003) 95-112. 
cursos de Brasil, uno de Chile - que aunque no es miembro del Mercosur, ha participado en todos los encuentros de biblioteconomía realizados -, uno de Paraguay y uno de Uruguay, a partir de la recopilación realizada en el Primer encuentro de los directores de los cursos superiores en Biblioteconomía de los países del Mercosur, realizado en 1996 y publicado en dos volúmenes por la Asociación Brasileña de Enseñanza de Biblioteconomía y Documentación (ABEBD). Para realizar el análisis comparativo, se construyeron dos matrices: una para los cursos de Brasil y otra para los demás países del Mercosur. A partir de ellas, se relacionaron las características comunes encontradas. Los indicadores para la caracterización de los diferentes currículos fueron dos: a) el objetivo de los cursos, buscando analizar el perfil profesional que cada curso pretende formar; y b) la organización curricular de los cursos, o sea, la manera en que los cursos organizan su estructura de conocimiento, el rol de las asignaturas que lo componen y su articulación con los objetivos propuestos, entendiendo por organización curricular la forma en la que los contenidos del curso se organizan. En el analisis de ambos indicadores se buscó explicitar, en lo posible, su fundamento teorico, es decir, la concepción del profesional subyacente a estos indicadores. El perfil de los docentes - titulación y régimen de trabajo (dedicación a la enseñanza, investigación y extensión) - y los cursos de educación continua para egresados, especialistas y pregraduados se estudiaron a partir de una encuesta enviada a treinta de Brasil, siete de Argentina, dos de Chile, una de Paraguay y otra de Uruguay.

\section{Nuevo orden mundial, educación superior y políticas públicas}

El nuevo orden mundial se puede caracterizar por la globalización de la ecoconomía, la emergencia de la Tercera Revolución Industrial, la intensificación de la competencia comercial en el mercado internacional y por el fin de la hegemonía comercial de los Estados Unidos y el surgimiento de bloques regionales capitalistas. La formación de megabloques trajo la necesidad de acuerdos económicos de integración. Sin embargo, esta integración extrapola las cuestiones económicas y políticas, e implica de manera suyacente la necesidad de internacionalización de sus sistemas de enseñanza superior, que pasan a desempeñar un papel de extrema relevancia en esta nueva coyuntura. La internacionalización supone un modelo de enseñanza superior sin fronteras nacionales, donde profesores y estudiantes tengan la posibilidad de producir y cambiar conocimientos, independientemente de su nacionalidad de origen. Esto supone modelos educacionales con currículos armonizados y cuya formación profesional tenga bases comunes.

En América Latina, la idea de la integración se debate hace mucho tiempo. El Mercosur - Mercado Común del Sur - objetiva la integración económica entre Argentina, Brasil, Uruguay y Paraguay. Tuvo su origen en 1980, cuando fueron firmados once acuerdos de cooperación entre Argentina y Brasil. En 1985, Brasil

Scire. $9: 1$ (en.-jun. 2003) 95-112. 
y Argentina firmaron la Declaración del Iguaçu, que abrió camino para una serie de tratados en el campo económico, que contribuyeron muchísimo para la formación del Mercosur en 1991. Esta declaración propició un proceso de integración entre Argentina y Brasil, que hasta ese momento mantenían relaciones difíciles, con periodos de rivalidad mezclados con tentativas de aproximación. En 1986, se aprobó el Programa de Integración y Cooperación Económica (PICE) entre Brasil y Argentina. Esos primeros tratados tenían un carácter de integración puramente económica, que buscaba resolver los problemas de esa naturaleza vividos por los dos países. Solamente a partir de marzo de 1991 esta integración avanzó un paso más con la firma del Tratado de Asunción, que incluía a Uruguay y Paraguay. Según Morosini (1998a, p. 28), el Mercosur "se constituye en la iniciativa internacional más importante en la última década de América del Sur y se propone realizar una profunda reestructuración política en esos países". Para Lampert (1998, p. 2) el Mercosur es una tentativa de varios países de América del Sur de organizarse, tras los largos años de dictadura, para resolver los problemas económicos y sociales que afectan a la vida del ciudadano sudamericano. El Mercado Común del Sur - Mercosur - fue creado en 29 de marzo de 1991, con el objetivo de ampliar las dimensiones de los mercados nacionales de Argentina, Brasil, Paraguay y Uruguay, y de promover la libre circulación de bienes y servicios y factores de producción en la región. Se presentó, sobre todo, como una perspectiva de vida más digna para las poblaciones de los países miembros (1).

La educación se mostró, desde el primer momento, como un elemento clave para la integración regional, al tiempo que podía contribuir de manera expresiva a la superación de las desigualdades regionales, la consolidación de la democracia y el desarrollo económico y social; pues a través de la educación se pueden superar las desigualdades regionales y promover el desarrollo. El Mercosur Educacional se desarrolló paralelamente al Tratado de Asunción y se responsabilizó de una serie de protocolos que aceleraron la maduración de las relaciones educacionales y el trabajo de la agencia. Uno de los más importantes es el Plan Trienal de Educación, firmado por los Ministros de la Educación de Argentina, Brasil, Paraguay y Uruguay, como países miembros, y de Chile y Bolivia, como países asociados, en 1995, y renovado para el Trienio 1998-2000. Además, el 20 de junio de 1996, se aprobó el documento Mercosur 2000: Desafios y metas para el Sector Educacional, que estableció como áreas prioritarias para la concentración de recursos y esfuerzos en el periodo 1997-2000 la renovación educacional, la evaluación educacional, la relación entre educación y trabajo, y la cooperación universitaria.

\section{La universidad en los países del Mercosur}

Del punto de vista político-económico, la evolución de los países del Mercosur a mediados de los 80 se caracteriza por dos procesos relacionados: la

Scire. $9: 1$ (en.-jun. 2003) 95-112. 
transición de regímenes militares a la democracia y la profunda crisis económica. Para otros países, la década de 80 representó una época de grandes cambios políticos, económicos y sociales. De los países del Mercosur, Paraguay fue uno de los últimos a promover reformas estructurales relacionadas con el proceso de reconstrucción democrática. Tras treinta y cinco años de régimen militar, el país inició en 1989 un periodo de transición democrática que hasta hoy no se ha consolidado. En 1998, fue promulgada la Ley General de Educación que dio nueva estructura y funcionamiento a la educación nacional. Uruguay tuvo una década de autoritarismo de 1973 a 1984. Después de 1985, Uruguay sufrió un periodo de transición política a la democracia, que nunca fue totalmente destruída, pues estaba bastante arraigada en la sociedad. A mediados de 1985, se normaliza la situación política y se aprueba la Ley General de Educación, que estableció los principios y objetivos desde el punto de vista de una sociedad democrática. En Chile, el régimen militar logró en 1980 la aprobación de una nueva Constitución Política del Estado que garantizase el programa de seguridad nacional y un marco económico neoliberal. La previsión social, el sistema de salud, las leyes laborales y el sistema de educación fueron objeto de profundos cambios en ambas dos líneas. Los sectores ideologizados por el gobierno militar propusieron un plan de acción para el sector universitario, que incluía una fuerte presión de las autoridades militares sobre el sector académico y una carencia de canales de participación, que afectaron la vida universitaria hasta el fin de la década de los 80. En Brasil, se inicia la apertura política a finales de la década de los 70 después de largos años de régimen militar. El marco jurídico de un nuevo estado fue promulgado en la Constitución de la República Federativa de Brasil, en octubre de 1988. Esta constitución muestra la ruptura con el ordenamiento constitucional anterior y el surgimiento jurídico de un nuevo estado. Argentina fue uno de los primeros países del Mercosur en iniciar su proceso de reformas estructurales en su modelo de desarrollo socio-económico. Con el regreso de la democracia en 1984, se inicia nuevo periodo en la vida del país, hoy conturbado por los graves desequilibrios económicos (2).

Por otra parte, aunque se identifiquen puntos comunes en los sistemas universitarios de los países del Mercosur, su diversa localización geográfica, las luchas económico-sociales propias de los países en desarrollo - signo de la inestabilidad político-democrática - , y la expansión de la educación superior privada, convierten la tarea de integración en un proceso desafiante; y, sin embargo, necesario para el proceso de integración regional en los aspectos sociales, económicos y políticos. Por otra parte, los objetivos o principios de una educación democrática están formalizados legalmente en los cinco países, y las estructuras educacionales determinadas por las respectivas legislaciones reúnen elementos fundamentales que favorecen el proceso de integración en curso. Los temas y problemas son bastantes comunes en el conjunto de los diferentes países. Sin

Scire. $9: 1$ (en.-jun. 2003) 95-112. 
embargo, las reformas siguen rumbos diferentes, condicionados en cada país por la coyuntura política y económica.

Entre las cuestiones comunes, destacan la expansión de la matrícula y el crecimiento del sector privado. En Uruguay, la Universidad de la República, la única universidad pública, centraliza prácticamente toda la enseñanza superior. Aparte hay otra universidad privada, dedicada a las Ciencias Humanas. Uruguay cuenta además con centros de formación de profesores y centros de enseñanza superior vinculados a sistemas universitarios de otros países. Paraguay, de la misma manera que Uruguay, concentra su sistema de enseñanza superior en la Universidad Nacional de Asunción (UNA) y en la privada Universidad Católica de Asunción (UCA). Posee también instituciones no universitarias que ofrecen cursos de corta duración. En Brasil y Chile la expansión del sector privado ha sido grande, a pesar de que no se haya derivado de una política propiamente dicha. En algunos casos, se produjo como consecuencia de una reforma universitaria, que actuó como un estímulo indirecto. Así pasó en Chile en 1967, y entre 1980 y 1982; y en Brasil en 1968. Además, se produce una coincidencia cronológica en lo que atañe a la expansión de los sistemas de educación superior. En todos ellos, con la excepción de Argentina, las matrículas crecieron de manera acelerada a fines de los años 60 e inicio de los 70. Brasil y Chile se distinguen por la existencia de una reforma universitaria en este periodo. En Brasil, la intervención del gobierno a través de la reforma se limitó al sector público, mediante la ampliación de la oferta de plazas y la creación de nuevas universidades federales. Sin embargo, por contraposición al alto índice de crecimiento del sector privado, el sector público se expandió poco. En Chile uno de los efectos de la reforma de 1967 fue la expansión del sector público, interrumpida en 1973 con la caída del gobierno democrático. Con la implantación del gobierno autoritario disminuyó drásticamente el número de matrículas. A fines de 1980, con una nueva reforma universitaria, se inauguró una segunda etapa de expansión. En Argentina, la expansión de las matrículas se produjo precozmente, coincidiendo con el primer periodo peronista, entre $1945 \mathrm{y}$ 1955, a causa principalmente de la política de egreso ilimitado.

En síntesis la expansión de los sistemas de educación superior en los países del Mercosur se produjo en dos momentos: el primero fue a fines de los años 60 e inicio de los 70, y el segundo ocurrió en los años 80 , coincidiendo con el proceso de descompresión de los regímenes autoritarios en Chile y, en menor escala, en Argentina.

\section{La formación profesional en Biblioteconomía en el Mercosur}

\subsection{Objetivos de la formación en Bibliotecología}

El análisis del primero de los indicadores seleccionados para efectuar la comparación - objetivos curriculares - ha permitido explicitar la concepción

Scire. $9: 1$ (en.-jun. 2003) 95-112. 
del profesional subyacente a estos indicadores; en especial, qué universidades situaban la investigación como elemento nuclear del perfil profesional buscado. En Argentina el programa que más enfatiza la investigación es el de la carrera de Bibliotecología y Documentación de la Facultad de Filosofía y Letras, uno de cuyos objetivos es "promover y favorecer la implementación de trabajos de investigación, especialmente entre docentes graduados y estudiantes, que aumenten el cuerpo de conocimientos relevantes para las teorías y prácticas de todas las profesiones de información." (ABEBD, v. 1, p. 6, 1996). Otros cursos priorizan también entre sus objetivos los conocimientos de metodología de la investigación. Detrás de esta concepción se detecta una filosofía docente orientada a graduar personas creativas, dispuestas a innovar ante problemas nuevos, y no simples repetidores de normas técnicas (3). La tabla I sintetiza las orientaciones docentes en Brasil. En Chile definen sus objetivos de forma muy genérica y amplia: "el cultivo y desarrollo de la ciencia biblioteconómica y de la información, así como la formación y el perfeccionamiento de los bibliotecarios del país a través de sus actividades de docencia, investigación y extensión” (ABEBD, v. 2, p. 205, 1996).

\begin{tabular}{|l|l|l|}
\hline Región & \multicolumn{2}{|l}{ Estados } \\
$\begin{array}{l}\text { Región } \\
\text { Norte }\end{array}$ & $\begin{array}{l}\text { Amazonas, Pará, } \\
\text { Maranhão }\end{array}$ & $\begin{array}{l}\text { Formación de profesionales que atiendan a las necesida- } \\
\text { des propias de la región, así como graduar bibliotecarios } \\
\text { capaces de desarrollar una práctica destinada a la realidad } \\
\text { social donde actúan. }\end{array}$ \\
\hline $\begin{array}{l}\text { Región } \\
\text { Nordeste }\end{array}$ & $\begin{array}{l}\text { Bahia,Ceará, Paraiba, } \\
\text { Pernambuco y Rio } \\
\text { Grande do Norte }\end{array}$ & $\begin{array}{l}\text { Cultura general, formación para bibliotecas populares, } \\
\text { públicas y bibliotecas escolares. }\end{array}$ \\
\hline $\begin{array}{l}\text { Región } \\
\text { Centro }\end{array}$ & $\begin{array}{l}\text { Goiás,Minas } \\
\text { Gerais,Distrito Federal }\end{array}$ & $\begin{array}{l}\text { Más diversificados, aunque sin perder su identidad regio- } \\
\text { nal. Colaborar en la creación de bibliotecas y centros de } \\
\text { documentación y realizar pesquisas en los varios dominios } \\
\text { de la cultura. }\end{array}$ \\
\hline $\begin{array}{l}\text { Región } \\
\text { Este }\end{array}$ & $\begin{array}{l}\text { Rio de Janeiro, Espirito } \\
\text { Santo }\end{array}$ & $\begin{array}{l}\text { Más amplios, dirigidos a lo social y para la sociedad en su } \\
\text { conjunto. Capacitar el futuro bibliotecario para asesorar } \\
\text { diferentes sectores profesionales, además de bibliotecas: } \\
\text { centros de documentación, institutos de investigación, tele- } \\
\text { visión, periódicos, bancos, marketing, oficinas de derecho, } \\
\text { clínicas médicas, etc. }\end{array}$ \\
\hline $\begin{array}{l}\text { Estado de } \\
\text { São Paulo }\end{array}$ & $\begin{array}{l}\text { Estado de São Paulo } \\
\text { Región Sur }\end{array}$ & $\begin{array}{l}\text { Pretende una formación más generalizada y destinada al } \\
\text { mundo empresarial. Formar profesionales destinados a } \\
\text { los centros de diseminación de la información en cadenas } \\
\text { y sistemas de información, sean bibliotecas, centros de } \\
\text { documentación o archivos. }\end{array}$ \\
\hline $\begin{array}{l}\text { Santa Catarina } \\
\text { Rio Grande do Sul }\end{array}$ & $\begin{array}{l}\text { Objetivos destinados a la formación, de modo que atienda } \\
\text { las necesidades del mercado. Graduar profesionales cons- } \\
\text { cientes, críticos y capaces de actuar como educadores y } \\
\text { agentes de transformación. }\end{array}$ \\
\hline
\end{tabular}

Tabla I. Objetivos docentes de los curricula brasileños

Scire. $9: 1$ (en.-jun. 2003) 95-112. 
Existe una preocupación por la formación filosófica y sociológica, específicamente en lo relativo a los fundamentos de la propia Biblioteconomía, además de "integrar grupos disciplinares entre sí en el desarrollo de proyectos de información a nivel institucional, nacional e internacional" (ABEBD, v. 2, 1996. p. 206). Esta preocupación acaba por caracterizar un currículo de naturaleza humanista. En Paraguay, al profesional graduado en Biblioteconomía se le denomina "administrador de información". El único curso existente forma profesionales en dos niveles: el intermedio - técnico en Biblioteconomía - y el de licenciatura. Los objetivos atañen tanto a la formación profesional como al desarrollo del espíritu de investigación. Uruguay, que gradúa licenciados en Biblioteconomía y tiene también la carrera de Archivología, tiene también como "objetivo fundamental la formación académica de los profesionales de la información" (ABEBD, v. 2, p. 259), y el fomento de la investigación es un objetivo fundamental, que se concreta en proyectos de investigación relacionados con las diversas áreas de interés de la Biblioteconomía.

Resumiendo, los objetivos de los diferentes cursos de Biblioteconomía de los países de América del Sur incluyen objetivos en la línea de proporcionar habilidades específicas de la profesión, desarrollar actividades de investigación, desarrollar las habilidades para formar un profesional abierto capaz de adaptarse a los retos de la globalización; y graduar ciudadanos éticos a través del desarrollo de la conciencia biblioteconómica.

\subsection{La organización curricular de los cursos en los diferentes países}

La organización curricular ha sido el otro indicador analizado, entendiendo por organización curricular la manera en la cual se articulan los contenidos de los cursos. Se han analizado los siguientes aspectos: la composición de los cursos en los diferentes ciclos de la formación del profesional; la organización de las asignaturas; la presencia de asignaturas de lenguas extranjeras, de prácticas, destinadas al estímulo de la investigación, y de tecnologías aplicadas; y la oferta de cursos de especialización y extensión. En todos los países se han producido numerosas reformas curriculares desde los años ochenta. En Brasil, la Asociación Brasileña de Enseñanza de Biblioteconomía y Documentación (ABEBD) incentivó, especialmente en la década de los ochenta e inicio de los noventa, constantes reformas curriculares, intentando acomodar los planes de estudio a las rápidas transformaciones tecnológicas y sociales de la sociedad globalizada.

Por lo general, las universidades argentinas ofrecen, además de la formación para la carrera de bibliotecario, el curso de licenciatura y profesorado en Biblioteconomía, que capacita al profesional para la dirección de unidades de información, docencia e investigación. En total se ofrecen cuatro modalidades: Bibliotecario escolar (curso de 2 años de duración), Bibliotecario-

Scire. $9: 1$ (en.-jun. 2003) 95-112. 
Documentalista (curso de 3 años), Bibliotecario-Profesor de Biblioteconomía (4 años) y Licenciado-Profesor investigador (5 años).

En Brasil, se han producido en las últimas décadas sucesivas reformas curriculares, todas dentro de un currículo mínimo que ha estado en vigor hasta 1996. La nueva Luz de Directrices y Bases de la Educación Nacional, Ley 9394/96, relativa a la Educación Superior, todavía en implantación, afirma en el artículo 53 del capítulo IV: "En el ejercicio de su autonomía se asegura a las universidades sin perjuicio de otras las siguientes atribuciones: [...] II. Fijar los currículos de sus cursos y programas, observadas las directrices generales pertinentes. III. Establecer planes, programas y proyectos de investigación científica, producción artística y actividades de extensión."

Los cursos de Brasil disponen, en su gran mayoría, de taller de informática, ofrecen el inglés instrumental y asignaturas de introducción a la investigación en su currículo. Algunos de ellos prevén trabajos de fin de curso o monográficos y asignaturas optativas.

Por su parte, en Chile se dio la última reestructuración curricular en 1990. La carrera tiene una duración de diez semestres. Con el propósito de garantizar la formación integral, el currículo contempla asignaturas generales, básicas y especializadas - Archivística, Biblioteconomía y Ciencia de la Información-, el inglés instrumental como lengua extranjera, y asignaturas destinadas a la formación investigadora, aunque no se exige monografía o trabajo de fin de carrera. Como sucede en Argentina, el currículo chileno contiene la asignatura "Biblioteca Escolar y Pública", un grupo de asignaturas con contenidos humanísticos, "Tecnologías de la información” y "Estudios y formación de usuarios".

En Paraguay, la última reformulación curricular se produjo en 1992, y la Escuela de Biblioteconomía se rebautizó Escuela de Biblioteconomía y Ciencia de la Información. El curso tiene dos modalidades: Técnico en Biblioteconomía (2 años) y Licenciado en Biblioteconomía y Ciencia de la Información (4 años). El currículo posee asignaturas destinadas específicamente a la profesionalización. Algunas son novedosas, como la de "Biblioteconomía Comparada", dónde se estudia la situación de la Biblioteconomía y Ciencia de la Información en el ámbito nacional e internacional, a través del método comparativo. No hay asignaturas destinadas a la formación investigadora. A ello no es ajeno el hecho de que, según las fuentes investigadas, "no existe un programa sistemático de desarrollo de investigaciones, ya que los docentes no tienen dedicación exclusiva" (ABEBD, v. 2, 1996, p. 258).

En Uruguay la Escuela de Biblioteconomía y Ciencia Afines gradúa el licenciados en Biblioteconomía, con un currículo compuesto de asignaturas técnico-profesionales y humanísticas como "Historia del Pensamiento Filosófico"

Scire. $9: 1$ (en.-jun. 2003) 95-112. 
y "Proceso Cultural de Uruguay". También se ofrece como modalidad de formación profesional la carrera de Archivología. Ambas carreras incluyen en sus currículos asignaturas de formación y práctica de la investigación. Las líneas de investigación se orientadas a temáticas diversas. Las asignaturas optativas también son contempladas en ambos currículos, y la escuela cuenta "con una unidad de Educación Permanente y Perfeccionamiento que tiene como objetivo la actualización y reciclaje de los egresos de ambas las carreras" (ABEBD, v. 2, 1996, p. 262). La preocupación más importante de ambas carreras es la inserción en el medio social y la conexión multidisciplinar con áreas afines con la finalidad de potenciar actividades cooperativas de integración (ABEBD, v. 2, 1996, p. 263).

Con excepción de Brasil, se observa que los currículos enfatizan poco las asignaturas destinadas a las nuevas tecnologías y a la informatización. Además, las prácticas, a pesar de tener objetivos definidos, no siempre son regladas, y existen pocas asignaturas destinadas a la investigación. La heterogeneidad de los currículos sugiere la necesidad de una adecuación mayor, especialmente en lo que concierne a las nuevas tecnologías y a las conquistas de la modernidad. En conclusión, los diferentes programas de los países del Mercosur presentan aspectos diferenciados, pero se detecta, de manera generalizada, un interés por mejorar la formación profesional, patente en el énfasis otorgado a la implantación de cursos de educación continua, la cualificación del cuerpo docente, y la adecuación curricular a las necesidades locales, nacionales y globales. Los contrastes y las semejanzas analizados permiten obtener una visión panorámica de las especificidades de cada curso.

\section{Formación docente}

La formación docente se estudió a partir de la encuesta señalada en la sección 1. Tras un test previo realizado a los coordinadores - coordinador y vicecoordinador - del curso de Biblioteconomía de la UNESP de Marília y las pertinentes modificaciones, las encuestas fueron enviadas en marzo de 1999 a los treinta cursos de Brasil, siete de Argentina, dos de Chile, uno de Uruguay y uno de Paraguay, en total cuarenta y uno. Hasta junio de 1999 se recibieron veintitrés encuestas debidamente rellenadas - diecisiete de Brasil, tres de Argentina, una de Chile, una de Uruguay y una de Paraguay - que constituyeron una muestra aceptable, puesto que todos los países quedaron representados en la debida proporción.

Los cursos fueron clasificados en dos grandes grupos: los de Brasil y los de los demás países del Mercosur, pues se consideró que se podía perder información si se consideraba un único grupo. Se les aplicaron el test de contraste de hipótesis denominado de diferencias entre proporciones (tabla II) y el test no paramétrico denominado $\chi^{2}$ (Chi-Cuadrado) (tablas III y IV). En la tabla II se presentan las puntuaciones para los diferentes objetivos de contratación de instituciones de

Scire. $9: 1$ (en.-jun. 2003) 95-112. 
Brasil y de los diferentes países del Mercosur. Obsérvese que las modalidades no son mutuamente excluyentes. Tras aplicar el test de la diferencia entre dos proporciones (estadístico z) se obtuvieron los siguientes resultados:

- Impartir clases y desarrollar investigación: Para esta categoría se obtuvo $\mathrm{z}=7,33$, que indica una diferencia muy significativa. Efectivamente, en Brasil el $62 \%$ de los docentes son contratados con dicho objetivo, frente al $23 \%$ en los demás países del Mercosur.

- Para impartir clases y actividades técnicas: Se obtuvo $\mathrm{z}=3,48$, que indica una diferencia significativa. Efectivamente, la proporción de docentes contratados para impartir clases y actividades técnicas es mayor en Brasil (el $28 \%$ ) que en los otros países (el 13\%).

- Solamente para investigación: Se obtuvo z=0, indicando que la situación en Brasil y el resto del Mercosur es semejante.

\begin{tabular}{|l|c|c|c|c|c|c|}
\hline Tipo de contrato & \multicolumn{3}{c}{ Brasil } & \multicolumn{3}{c|}{ Resto } \\
\hline Solamente para impartir clases & 61 & $27 \%$ & 97 & $66 \%$ & 158 & $42 \%$ \\
\hline Impartir clases y desarrollar investigación & 141 & $62 \%$ & 33 & $23 \%$ & 174 & $47 \%$ \\
\hline Impartir clases y actividades técnicas & 63 & $28 \%$ & 19 & $13 \%$ & 82 & $22 \%$ \\
\hline Solamente para investigación & 5 & $2 \%$ & 3 & $2 \%$ & 8 & $2 \%$ \\
\hline Otras (administrativas) & 5 & $2 \%$ & 5 & $3 \%$ & 10 & $3 \%$ \\
\hline Total de docentes & 227 & $100 \%$ & 146 & $100 \%$ & 373 & $100 \%$ \\
\hline
\end{tabular}

Tabla II, Frecuencia y tanto por ciento de los objetivos de contratación de los docentes. El mismo docente fue incluido, al mismo tiempo, en las categorías 2 y 3.

Brasil presenta mayor porcentaje de docentes contratados para impartir clases y desarrollar investigación - el 62\% - que los demás países del Mercosur -el 23\% - , mientras que en estos últimos es mayor el porcentaje de docentes contratados para impartir tan solo clases -el 66\%-. Por medio de la aplicación del test $\chi^{2}$ (Chi-cuadrado), se verificó si existía relación entre los regímenes de contratación de profesores entre las instituciones de Brasil y los demás países del Mercosur. Se obtuvo $\chi^{2}=130,92$ y $\chi c^{2}=5,99$, con dos grados de libertad y 0,05 de significancia. Como $\chi c^{2}<\chi^{2}$, se concluye que existe una alta asociación entre las variables. Efectivamente, en Brasil hay mayor tendencia a contratar a tiempo completo (más de 30 horas) mientras que en los demás países la tendencia es a contratar a tiempo parcial (menos de 30 horas). En Brasil, el 17\% de los docentes son contratados a tiempo completo; en los demás países del Mercosur, este porcentaje cae hasta el $13 \%$, pues la gran mayoría de los docentes es contratada a tiempo parcial.

Scire. $9: 1$ (en.-jun. 2003) 95-112. 


\begin{tabular}{|l|c|c|c|c|c|c|}
\hline Modalidad de contrato & \multicolumn{2}{c}{ Brasil } & \multicolumn{3}{c|}{ Resto } & \multicolumn{3}{c|}{ Total } \\
\hline A tiempo completo ( $\geq 30$ horas) & 162 & $71 \%$ & 19 & $13 \%$ & 181 & $49 \%$ \\
\hline A tiempo parcial (<30 horas) & 50 & $22 \%$ & 118 & $81 \%$ & 168 & $45 \%$ \\
\hline Profesor contratado eventualmente & 15 & $7 \%$ & 8 & $5 \%$ & 23 & $6 \%$ \\
\hline Profesor invitado & - & - & 1 & $1 \%$ & 1 & $0 \%$ \\
\hline TOTAL DE DOCENTES & 227 & $100 \%$ & 146 & $100 \%$ & 373 & $100 \%$ \\
\hline
\end{tabular}

Tabla III. Frecuencia y porcentaje de los tipos de contrato de los docentes.

Las tablas IV y V presentan el perfil de titulación de los docentes de Brasil y del resto de los países. La tabla IV presenta la frecuencia de docentes contratados en los 17 cursos estudiados. El valor obtenido en el test $\chi^{2}$ fue 93,78, lo cual indica una fuerte asociación entre la titulación de los docentes y su lugar de trabajo (Brasil y el resto de los países); es decir, pone de manifiesto que el perfil en Brasil es notablemente distinto del de los demás países. Los datos de la tabla $\mathrm{V}$ muestran que Brasil presenta un cuerpo docente con un alto porcentaje de másters - el 43,14\% - . En los otros países del Mercosur, el título final de la mayor parte de los docentes es el grado: De 127 docentes, 119, esto es el 93\%, son graduados -incluyendo los que no han terminado su especialización o máster-, mientras que sólo el $4 \%$ tiene título de máster.

\begin{tabular}{|l|c|c|c|c|c|c|}
\hline Titulación & \multicolumn{2}{c}{ Brasil } & \multicolumn{2}{c}{ Resto } & \multicolumn{2}{c|}{ Total } \\
\hline Graduación (Licenciatura) & 20 & $9 \%$ & 80 & $55 \%$ & 100 & $27 \%$ \\
\hline Graduación con especialización & 48 & $22 \%$ & 33 & $23 \%$ & 81 & $22 \%$ \\
\hline Máster no concluído & 19 & $8 \%$ & 6 & $4 \%$ & 25 & $7 \%$ \\
\hline Máster concluido & 64 & $28 \%$ & 5 & $3 \%$ & 69 & $18 \%$ \\
\hline Doctorado no concluido & 29 & $13 \%$ & 1 & $1 \%$ & 30 & $8 \%$ \\
\hline Doctorado concluido & 31 & $14 \%$ & 2 & $1 \%$ & 33 & $9 \%$ \\
\hline Postdoctorado & 3 & $1 \%$ & - & - & - & - \\
\hline Sin observación & 13 & $6 \%$ & 19 & $13 \%$ & 32 & $9 \%$ \\
\hline TOTAL DE DOCENTES & 227 & $100 \%$ & 146 & $100 \%$ & 373 & $100 \%$ \\
\hline
\end{tabular}

Tabla IV. Frecuencia y porcentaje de la titulación de los docentes.

Estos datos, aunque parciales, proporcionan una visión panorámica de los países del Mercosur, y ponen de manifiesto una de las barreras a superar de cara a la armonización curricular. Una enseñanza de calidad, donde la investigación y la

Scire. $9: 1$ (en.-jun. 2003) 95-112. 
producción científica tengan pleno espacio de desarrollo, exige docentes contratados para investigar, a tiempo completo y con titulación adecuada, no solo para impartir clases. Cabe señalar que Brasil y Argentina tienen una presencia significativa en la producción científica del Mercosur. Brasil tiene una posición privilegiada, merced a las inversiones de los años 70 y 80. Según Lambert (Lambert, 1998, p. 7), el crecimiento de la producción científica en Brasil en la década de los 70 fue espectacular: entre 1973 y 1980, la participación brasileña en el ISI (Institute for Scientific Information) prácticamente se triplicó.

\begin{tabular}{|c|c|c|}
\hline Titulación & Brasil & Resto \\
\hline Graduación & 87 & 119 \\
\hline Máster concluido & 93 & 6 \\
\hline Doctorado concluido & 34 & 2 \\
\hline Total & 214 & 127 \\
\hline
\end{tabular}

Tabla V. Titulación de los docentes (resumida).

\section{Análisis de los cursos de formación continua}

La oferta de cursos de formación continua se analizó a partir de una serie de tres años - 1996, 1997 y 1998 - . Tras la lectura de cada curso analizado, se identificó el asunto y se clasificó según dos criterios: uno geográfico, separando los cursos en dos grupos, los de Brasil y los de los demás países del Mercosur; y otro temático, de acuerdo a las seis grandes áreas del conocimiento biblioteconómico consignadas en el I Encuentro de Docentes de Biblioteconomía y Ciencia de la Información de los países del Mercosur, que tuvo lugar en Buenos Aires en 1997, y que son las siguientes: área 1, fundamentos teóricos; área 2, organización de la información; área 3, recursos y servicios de información; área 4, tecnología de la información; área 5, gestión de unidades de información; y área 6, investigación. Estas seis grandes áreas constituyeron los núcleos alrededor de las cuales se reunieron los docentes participantes. Se eligió esta clasificación por su compatibilidad con las áreas definidas por las directrices formuladas por la Secretaria de Educação Superior del Ministério da Educação del Brasil en junio de 1999.

Los diecisiete centros de Brasil y los seis de los demás países del Mercosur que enviaron sus respuestas ofrecían cursos de formación continua en las seis áreas. Para su clasificación temática se contó con la asesoría de un profesional del área de Biblioteconomía. Se registró una frecuencia grande de cursos en el área 5 - Gestión en Unidades de Información-, quizá porque es uno de los ejes temáticos que más ha preocupado a los profesionales y académicos del área. Le sigue el área 1 -Fundamentos Teóricos de Biblioteconomía y Ciencia de

Scire. $9: 1$ (en.-jun. 2003) 95-112. 
la Información -, tema relevante por la necesidad de sustentar teóricamente el área. Después está el área 4 -Tecnologías de la Información-, también muy relevante. Se observó que en las áreas 1, 4 y 5 había mayor frecuencia de cursos ofrecidos que en Brasil, especialmente en el área 4 - cursos de Microisis - y en el área 5 - cursos sobre Gestión y Organización de la Información-, destacando también los cursos sobre Marketing. En el área de investigación científica -área 6- se ofrecían muchos cursos, lo que sugiere un gran interés e incluso necesidad de formación en este área.

Respecto a la modalidad de los cursos, la gran parte fueron de extensión tanto en Brasil como en los demás países del Mercosur. La carga horaria varió grandemente, desde dos horas para las conferencias hasta 840 horas para los cursos de especialización. En cuanto al número de inscritos, se verificó una relación entre la amplitud del tema del curso y el número de interesados: los cursos más específicos presentaron menor inscripción que los de mayor amplitud. Así, cursos como el de "Conservación de Fondos" tuvo muchos menos inscritos que el dedicado a "La sociedad global y la gestión de la información", mucho más amplio temáticamente y dedicado a aspectos sociales y políticos.

Con relación a la titulación de los docentes que impartieron los cursos de Educación continua en Brasil, la gran mayoría - aproximadamente el 60\%tenía el título de máster, el $21 \%$ de doctor, y el 19\% de licenciado. En los demás países del Mercosur, los profesores eran mayoritariamente licenciados (50\%), seguidos de los doctores (31\%) y maestros (19\%). En general, eran invitados de otros países, especialmente de Brasil, y pertenecían a diferentes áreas, no siempre conectadas a la Biblioteconomía y la Ciencia de la Información. Generalmente, los docentes eran especialistas en los temas de los cursos, con algunas excepciones en los cursos cuyos temas estaban más alejados de las áreas de actuación de los docentes. Los profesionales invitados con más frecuencia fueron profesores, universitarios, bibliotecarios, archiveros, museólogos, representantes de sindicatos, de empresas y consultorías de empresas, educadores, profesionales de la informática, y representantes de instituciones ligadas a la Ciencia y la Tecnología.

\section{Consideraciones finales}

Es evidente que la integración educacional en el área de Biblioteconomía y Ciencia de la Información en los países del Mercosur es ya una realidad que avanza rápidamente, de modo que es posible considerar las acciones educacionales como un ámbito propio y distinguible de las acciones económicas y políticas, aunque el proceso de integración en el ámbito de la educación se haya iniciado a partir de las relaciones comerciales. Aunque actualmente todos los países comparten los objetivos y principios de una educación de carácter democrático (4), cada uno de ellos se encuentra en un momento diferente en lo que concierne al

Scire. $9: 1$ (en.-jun. 2003) 95-112. 
desarrollo y a la implementación de políticas de enseñanza superior, hecho que se refleja también en la enseñanza de la Biblioteconomía. La integración exige la minimización de los puntos de diferenciación, no tanto en el sentido de nivelarlos, sino de encontrar puntos de identidad entre los varios sistemas de educación superior, tanto en lo que concierne a la organización y objetivos de los cursos, como en lo relacionado con la propia estructuración curricular. Y es en este sentido en el que se detectan a corto plazo desafíos para la integración, que se apuntan aquí para favorecer el fortalecimiento de este proceso, para que conduzca a la formación de un profesional adecuado.

La primera cuestión es la validez de los diplomas universitarios. La libre circulación de profesionales está estrechamente conectada al reconocimiento y a la validez de los diplomas universitarios. El desarrollo del proceso de convergencia educacional, enmarcado en el Plan Trienal de Educación y del Mercosur 2000 - los dos documentos que contienen todos los programas educacionales previstos para los próximos años - debe consolidarse de forma más eficaz. Los protocolos de enseñanza superior acordados desde 1996, en lo que se refiere a los estudios de postgrado, la formación de recursos humanos para el postgrado, el reconocimiento de títulos y grados universitarios, deberían transformarse en normas jurídicas, para lo cual es necesario que las entidades relevantes de las diferentes áreas del conocimiento se posicionen al respecto.

Otra cuestión es la relativa al desarrollo de los conceptos pedagógicos comunes, que son los pilares sobre los cuales construir la integración. El primer paso de la armonización curricular requiere propuestas pedagógicas comunes para la enseñanza en los diferentes países. Un aspecto fundamental que se debe destacar es la necesidad de incrementar el papel de la investigación en la enseñanza de pregrado, fortaleciéndola como elemento de intervención en el cotidiano, garantizando un titulado apto para desafiar circunstancias diversas en el mercado de trabajo, capaz de actuar en diversas instancias y en un universo geográfico más amplio. Se trata, en definitiva, de desarrollar una actitud metodológica en la que la investigación fundamente la enseñanza, convirtiéndola en un proceso que vaya más allá de la simple transmisión de conocimiento ya existente, capacitando para la creación de conocimiento nuevo, más allá de la simple copia. Pero eso requiere un profesorado adecuado, y la presente investigación ha mostrado que la mayor parte de las instituciones de enseñanza superior, con la excepción de las universidades de Brasil, contrata profesores por horas, solamente dedicados a la enseñanza. Por ello, son necesarias políticas de enseñanza que incentiven el incremento de la titulación y la investigación, y se orienten a la contratación de docentes a tiempo completo, pues una de las tareas de la universidad es articular enseñanza e investigación.

Otro aspecto que parece fundamental, pensando en la formación del profesional bibliotecario que actuará en los próximos años, es la necesidad de formación

Scire. $9: 1$ (en.-jun. 2003) 95-112. 
continua. Solamente una parte de la formación profesional se da en los estudios de grado. Por ello, es fundamental la educación continua o permanente como base para que el profesional pueda actualizarse y amoldarse a los constantes cambios que se producen en el mundo de la tecnología y de la información. En esta época, ningún currículo universitario suministra todo lo que el profesional necesita, así como ningún curso contiene el saber necesario para asegurar una carrera de éxito. La educación continua se encuentra en proceso de consolidación en el ámbito del Mercosur, aunque está más desarrollada en el ámbito nacional. Obsérvese, por otra parte, que los encuentros, conferencias, simposios y cursos realizados por las diferentes instituciones facilitan la integración, pues constituyen momentos de reunión en los que los profesionales de las diferentes instituciones y países contemplan temáticas y problemas comunes.

Para finalizar este trabajo, es necesario observar que el Mercosur Educacional, especialmente en lo que concierne a la educación superior, no se propone resolver los grandes problemas educativos de América del Sur, pero inserta la educación en el proceso de integración regional, delineando la construcción de un nuevo modelo de sistema de educación superior. Un modelo que transciende los límites de las naciones, que posibilita la movilidad de las personas, facilita la integración económica, política y social, y la formación de profesionales ágiles para actuar fuera de los estrechos límites nacionales, buscando la mejora de la calidad de vida de la población. En definitiva, y según afirma Valentín (2000, p. 11):[...] la integración entre los países del Mercosur, en el área de Biblioteconomía y Documentación, es un hecho, una realidad. [...] Más aún, según la autora, "el mercado común, de una forma general, busca acciones que fortalezcan la profesión en el ámbito de su influencia económica. El Mercosur es un ejemplo de eso y de cómo la integración promueve el crecimiento y la consolidación de un área" (ibidem).

\section{Notas}

(1) Consiste en un gran conjunto de acuerdos entre los Estados-Partes que incluye la libre circulación de bienes, servicios y factores de producción entre los países a través de la eliminación de las leyes aduaneras y de las tarifas de circulación de mercancías, el establecimiento de una tarifa externa común y la adopción de una política comercial común con otros Estados, la coodinación de políticas macroeconómicas y sectoriales del comercio exterior, agrícola, industrial, fiscal, monetaria, cambial y de capitales, de servicios, aduaneras, de transportes y comunicaciones y en todas las otras áreas que se acuerden, así como el compromiso de los Estados-Partes de armonizar sus legislaciones en las áreas pertinentes para consolidar el proceso de integración.

(2) En Argentina, la educación superior está dividida en dos sectores: el universitario, impartido por las universidades públicas y privadas, y el terciario, constituido por cursos de corta duración, impartido en establecimientos de enseñanza. El sistema de enseñanza superior de Brasil se reparte entre las universidades públicas (municipales, estatales y federales) y privadas, con gran predominio cuantitativo de éstas últimas.

Scire. $9: 1$ (en.-jun. 2003) 95-112. 
(3) Por otro lado, estudios como los de la Universidad de Missiones denuncian el escaso desarrollo de actividades de investigación (ABEBD, v. 1, p. 282). En esta misma universidad, uno de los objetivos para optimizar la enseñanza es "mejorar la formación del cuerpo docente mediante la realización de estudios de postgrado y visitas a Centros de Excelencia" (ABEBD, v. 1, p. 282).

(4) La integración del Mercosur fortalece a los países para el enfrentamiento a la hegemonía de los países americanos y europeos. El proceso de integración educacional, al abarcar diferentes culturas, implicará complejos cambios en las estructuras tradicionales, a la vez que exigirá gran flexibilidad por parte de los países miembros para afrontar la previsible ampliación de la movilidad. En este aspecto, Brasil tiene un papel fundamental, toda vez que posee el sistema de postgrado más desarrollado y el sistema de enseñanza superior más complejo, debido a su extensión territorial.

\section{Referencias bibliográficas}

ABEBD (1996). Encontro dos dirigentes dos cursos superiores de Biblioteconomia do Mercosul: a formação profissional em Biblioteconomia no Mercosul. Porto Alegre, 1996.

Adamo, A. C.; Rodrigues,C. (1993). Analisis y recomendaciones en la formación de los profesionales de la información. // Reunión Nacional de Bibliotecarios: El futuro de la biblioteca en la sociedad del futuro. Buenos Aires, 1993. 27.

Barber, E. (1996). Carrera de bibliotecología y Documentación. // A Formação em Biblioteconomia no Mercosul. Porto Alegre, 1996. Vol.1. Não publicado.

Brasil. Resolução CEF n8, de 29 de outubro de 1982. Fixa os mínimos de conteúdo e duração do curso de Biblioteconomia. // Diário Oficial da União. Brasília. (8 nov 1982) Seção I, p.20.

Brasil. Parecer CFE n ${ }^{\circ} 460$, de $1^{\circ}$ de outubro de 1982 - Fixação dos mínimos de conteúdo e duração do curso de Biblioteconomia. // Revista Documenta. 262 (set. 1982) 72-82.

Brasil. Lei n 9.394/96. Lei Diretrizes e Bases. Sin publicar.

Brasil. Ministério da Educação e Cultura. Departamento de Políticas do Ensino Superior. Proposta de diretrizes curriculares. URL: <http://www.mec.gov.br>.

Características y objetivos de la carrera. Universidad de Buenos Aires. URL: <http:// www.rima.pucsp.br>. Consultado: 1999- 04-21.

Casassus, J. (1995). Concertación e alianzas en educación. // Flacso. Es posible concertar las políticas educativas?. Buenos Aires: Miño y Dávila, 1995. 17-40.

Diretrizes Curriculares Nacionais dos Cursos de Biblioteconomia. Ministério da Educação. Conselho Nacional de educação. URL: <http://www.mec.gov.br>. Consultado 2001-08-17.

Eisemon, T. O. (1995). Reformong Higher Education Sistems. URL: <http:// www.wordlbank.org/backgrnd/rhesys2.html>. Consultado: 1995-02-26

Encuentro de directores de los cursos superiores de bibliotecología del Mercosur, 2; encuentro de docentes de bibliotecología y ciencia de la información del Mercosur, 1,1997, Buenos Aires. Acuerdos y recomendaciones. Buenos Aires: Universidad de Buenos Aires, Departamento de Bibliotecología y Documentación, 1997.

Fernández, S. M. (1979). La enseñanza de la Bibliotecología en la Facultad de Filosofía y Letras. Bibliotecología y Documentación, Buenos Aires, 1979. 2, 5-30,

Scire. $9: 1$ (en.-jun. 2003) 95-112. 
Guimarães, J. A. C. (1998). III Encuentro de Directores y II Encuentro de Docentes de Bibliotecología del Mercosul. Santiago do Chile, 1998.

Relatório de afastamento. Sin publicar.

Jaguaribe, H. (s. f.). Mercosul: problemática factual e institucional. URL: <http:// www.adenaure.com.br>. Consultado: 2001-03-19.

Kent, R. (1996). Los temas críticos de la educación superior en América Latina: estudios comparativos. México: Fondo de Cultura Económica, 1996.

Lampert, E. (1998). Educação e Mercosul: desafios e perspectivas. // Revista da Faculdade de Educação. 24:2 (São Paulo, jul/dez. 1998). URL: <http://www.scielo.br>. Consultado: 2001-10-24.

Mason, R. O. (1990). What is an information professional? // Journal of education for library and information science. 31:2 ( Jan. 1990), 122-138.

Maturana, T. I. (1998). Formación de recursos humanos en el area de la información en el Mercosur. // Encuentro de Directores, 3; Encuentro de Docentes de las Escuelas de Bibliotecología y Ciencia de la Información del Mercosur, 2. Santiago, Chile, 1998.

Mercosul 2000. (Texto Oficial). URL: <http://www.mwc.gov.br/mercosur/default.htm>. Consultado: 1999-04-21.

Morosini, M. C. (1998a). Universidade no Mercosul. 2. ed. São Paulo: Cortez, 1998.

Morosini, M. C. (1998b). Mercosul/Mercosur: políticas e ações universitárias. Campinas, São Paulo: Autores Associados, 1998.

Morosini, M. C. (1997). Internacionalização de sistemas universitários: o Mercosul. // Sguissardi, V.: Silva Jr., J. R. Políticas públicas para a educação superior. Piracicaba: Editora UNIMEP, 1997. 225-254.

Santos, J. P. (1997). O ensino de Biblioteconomia no Brasil: propostas de integração e harmonização curricular. São Paulo: APB, 1997. (Ensaios APB, 41).

Saraiva, J.F.S. (1997). Mercosul: interação ou desenvolvimento regional?. Brasília: UNB, 1997. Sin publicar.

Sguissardi, V.; Silva Jr., J. R. (Org.) (1997). Políticas públicas para a educação superior. Piracicaba: Editora UNIMEP, 1997.

Sguissardi, V.; Silva Jr., J. R. (Org.) (2001). Novas faces da educação superior no Brasil. São Paulo: Cortez, 2001.

Sistema de informação em educação no Mercosul. URL: < http://www.mwc.gov.br/ mercosul/default.htm >. Consultado : 1999-04-21

Tratado de Assunção [s.n.t.].

Valentim, M. P. (2000). Profissionais da informação: formação, perfil e atuação professional. São Paulo: Polis, 2000.

Zibas, D. M. L.; Franco, M. L. P. B.; Warde, M. J. (Org.) (1997). Globalização e políticas educacionais na América Latina. // Cadernos de Investigación. São Paulo, 100 (1997).

Werthein, J. (1998). A universidade no século 21. // Folha de S. Paulo. (6 out. 1998) 3.

World Bank (task force). Higher education en developing countries: peril and promise. Washington D.C.: World Bank. URL: <http://worldbank.org>. Consultado: 2001-09-01. 